\title{
Urinary Oligosaccharide Excretion in Nephrotic Syndrome
}

\author{
YUKITOSHI TAKAHASHI, SHIROU OMIYA, YUTAKA NAKAMURA, NAOMI KONDO, AND \\ TADAO ORII \\ Department of Pediatrics, Gifu University School of Medicine, Gifu, Japan
}

\begin{abstract}
Urinary oligosaccharides from nephrotic syndrome patients were investigated by Bio-Gel P-4 column chromatography and methylation analysis to clarify the electrostatic defects and size barrier defects in the glomerular basement membrane of nephrotic syndrome patients. The levels of urinary oligosaccharides $(\mathrm{nmol} / \mathrm{mg}$ creatinine) in nephrotic syndrome patients were about 25 times the levels in controls. The ratio of neutral oligosaccharides to sialyl oligosaccharides was about $1: 1$ in the urine of nephrotic syndrome patients. The levels of neutral oligosaccharides in the urine of nephrotic syndrome patients were about 13 times that of controls. The proportion of trisialylated oligosaccharides in the urine of nephrotic syndrome patients was higher than that in galactosialidosis patients. These findings suggest that electrostatic defects and size barrier defects in the glomerular basement membrane might contribute to the characteristic oligosacchariduria of nephrotic syndrome patients. In addition, the structures of 11 different kinds of oligosaccharides that have not been previously reported were identified in the urine of nephrotic syndrome patients. (Pediatr Res 34: 429-434, 1993)
\end{abstract}

Abbreviations

MCNS, minimal change nephrotic syndrome GBM, glomerular basement membrane GC/MS, gas chromatograph/mass spectrometer

Nephrotic syndrome is characterized by hypoproteinemia and edema that result from severe lasting proteinuria. It is generally accepted that the proteinuria of glomerular diseases arises from dysfunction of the GBM that has both size-selective and chargeselective properties (1-5). Especially in MCNS, an electrostatic defect in the GBM has been regarded as a main determinant of proteinuria $(6,7)$. This electrostatic defect could be produced by the removal of heparan sulfate in the GBM (8).

Oligosaccharidosis patients excrete large amounts of urinary oligosaccharides resulting from incomplete degradation of $N$ glycosidically linked oligosaccharides within cells, although renal functions are not impaired (9). Normal subjects also excrete small amounts of urinary oligosaccharides $(10,11)$. These oligosaccharides originating from intracellular degradation of complex-type oligosaccharides are excreted into the urine of normal subjects and oligosaccharidosis patients despite the normal restrictive permeability properties of the GBM.

To investigate the dysfunction of the restrictive permeability properties in the GBM of nephrotic syndrome patients, we

Received September 9, 1992; accepted May 13, 1993.

Correspondence: Dr. Yukitoshi Takahashi, Department of Pediatrics, Shizuoka Higashi Hospital, 886 Urushiyama. Shizuoka 420. Japan. analyzed the urinary oligosaccharides of nephrotic syndrome patients.

\section{MATERIALS AND METHODS}

Patients. Clinical features and laboratory data of five children with nephrotic syndrome are shown in Table 1. Patients 1 and 2 were in acute onset stages, and patients 3,4 , and 5 were in chronic prolonged stages when urine was collected. All patients manifested characteristic features of nephrotic syndrome (urinary protein $>3.5 \mathrm{~g} / \mathrm{d}$; serum total protein $<6.0 \mathrm{~g} / \mathrm{dL}$; decreased level of $\mathrm{CH} 50$; negative test findings for rheumatoid arthritis, $\mathrm{HBs} \mathrm{Ag}$, and antinuclear antibody and hypercholesterolemia) during the acute stage. Patients 1 and 2 enjoyed remissions for about $5 \mathrm{y}$, and the proteinuria of patients 3,4 , and 5 has diminished gradually. None of the patients showed neurologic signs, mental retardation, or coarse faces. Patients $1,2,3$, and 4 seemed to suffer from MCNS, and patient 5 suffered from Henoch-Schönlein purpura nephritis, with histologic findings of III a.

Urine specimens and exoglycosidase. Urine specimens were collected from the five patients with nephrotic syndrome (acute stage, two patients; chronic stage, three patients), and were frozen and stored at $-25^{\circ} \mathrm{C}$ before use. The exoglycosidases used were Arthrobacter ureafacience $\alpha$-neuraminidase, jack bean $\beta$-galactosidase, jack bean $\beta$ - $N$-acetyl hexosaminidase, jack bean $\alpha$ mannosidase, and snail $\beta$-mannosidase. Glucose oligomers were obtained by partial acid hydrolysis of dextran as described in a previous paper (12)

Preparation and labeling of oligosaccharide fractions. Urine containing $10 \mathrm{mg}$ of creatinine was concentrated to $3 \mathrm{~mL}$ and subjected to Bio Gel P-4 (minus 400 mesh, Bio-Rad Laboratories, Richmond, CA) column chromatography and descending paper chromatography with solvent I (n-buthanol:ethanol:water, 4:1:1) to prepare oligosaccharide fractions (13). Mixtures of the oligosaccharide fractions and 2-deoxyribose (external standards) were reduced with $\mathrm{NaB}^{3} \mathrm{H}_{4}(25 \mathrm{mCi}, 341.0 \mathrm{mCi} / \mathrm{mmol}$, New England Nuclear, Boston, MA) by routine methods (14) and subjected to descending paper chromatography with solvent I to separate tritiated external standards. Because the labeling efficiency (cpm/ $\mathrm{nmol}$ ) was calculated from the radioactivity of tritiated external standards, the concentrations of urinary oligosaccharides were determined.

Tritiated oligosaccharides were subjected to high-voltage paper electrophoresis using $\mathrm{pH} 5.4$ pyridine/acetate buffer to separate neutral and acidic oligosaccharides (13). On the basis of the position to which the bromphenol blue marker migrated during this electrophoresis, acidic oligosaccharides were divided into three fractions. These acidic oligosaccharides could be desialylated by $\alpha$-neuraminidase digestion. Therefore, the three fractions were considered to be monosialylated oligosaccharides, disialylated oligosaccharides, and trisialylated oligosaccharides.

Neutral oligosaccharides were subjected, with glucose oligomer, to Bio-Gel P-4 column chromatography performed under 
Table 1. Clinical features and laboratory data of nephrotic syndrome patients*

\begin{tabular}{|c|c|c|c|c|c|}
\hline & \multicolumn{5}{|c|}{ Patient } \\
\hline & 1 & 2 & 3 & 4 & 5 \\
\hline Age at onset $(\mathrm{yr})$ & 4 & 9 & 4 & 4 & 12 \\
\hline Age at examination $(\mathrm{yr})$ & 4 & 9 & 5 & 5 & 15 \\
\hline Stage & Acute & Acute & Chronic & Chronic & Chronic \\
\hline Neurologic signs & & & & & \\
\hline Serum total protein $(\mathrm{g} / \mathrm{dL})$ & 4.1 & 4.3 & 5.2 & 5.3 & 6.7 \\
\hline Urinary protein $(\mathrm{g} / \mathrm{d})$ & 6.4 & 5.8 & 1.0 & 2.0 & 1.7 \\
\hline Treatment & Pred & Pred & Pred, pulse & Pred & Dipyr \\
\hline
\end{tabular}

* Pred, prednisolone: Dipyr, dipyridamole; pulse, methyl prednisolone pulse therapy.

special conditions (12) and were fractionated according to glucose units. A few subfractions from neutral oligosaccharides were subsequently subjected to descending paper chromatography with solvent II (ethylacetate:pyridine:acetic acid:water, 5:5:1:3) to get neutral oligosaccharide subfractions according to the isomerism.

Structural analysis of neutral oligosaccharides. The sequence of monosaccharides in each neutral oligosaccharide chain was determined by sequential digestions using exoglycosidase and repeating Bio-Gel P-4 column chromatographies under the special conditions $(12,13,15)$. The location of the glycosidic linkage between each monosaccharide in the neutral oligosaccharide chain was determined by methylation analysis and GC/MS analysis $(13,16)$.

Structural analysis of sialyl oligosaccharides. Three sialyl oligosaccharide fractions, isolated by high-voltage paper electrophoresis, were incubated with $A$. ureafacience $\alpha$-neuraminidase (0.2 unit) and $0.1 \mathrm{M}, \mathrm{pH} 5.0$ acetate buffer $(150 \mu \mathrm{L})$ at $37^{\circ} \mathrm{C}$ for $48 \mathrm{~h}$ to cleave sialic acid at the nonreducing terminal. The reaction solution was subjected to high-voltage paper electrophoresis using $\mathrm{pH} 5.4$ pyridine/acetate buffer to separate the neutralized oligosaccharides. The neutralized oligosaccharides were fractionated by Bio-Gel P-4 column chromatography under the special conditions (12) and descending paper chromatography using solvent II. The sequence of monosaccharides in each neutralized oligosaccharide chain was determined by the same methods as in the analysis of neutral oligosaccharides (13). The location of the glycosidic linkage between each monosaccharide in the sialyl oligosaccharide chain also was determined by methylation analysis and GC/MS analysis $(13,16)$.

\section{RESULTS}

Control. The amount of urinary oligosaccharides in the control urine $(n=6)$ was $2.6 \pm 0.5 \mathrm{nmol} / \mathrm{mg}$ creatinine. Urinary oligosaccharides consisted of neutral oligosaccharides that were eluted at less than five glucose units.

Nephrotic syndrome. The amounts of oligosaccharides excreted in the urine of nephrotic syndrome patients are shown in Table 2.

Urinary oligosaccharides were separated into three sialyl oligosaccharide fractions and a neutral oligosaccharide fraction by high-voltage paper electrophoresis. The amounts of each sialyl and neutral oligosaccharides are shown in Table 2 . The quanti- tative differences of each oligosaccharide fraction between acute stage and chronic stage patients were not statistically significant. The ratios of neutral oligosaccharides to sialyl oligosaccharides were $0.89 \pm 0.08$ in acute stage patients $(n=2)$ and $1.26 \pm 0.47$ in chronic stage patients $(n=3)$.

The neutral oligosaccharides were separated into seven subfractions (I through VII) by the Bio-Gel P-4 column chromatography (12) as shown in Figure 1. The oligosaccharide contents of the seven subfractions are presented in Table 3 . In most subfractions, the differences of oligosaccharide content between acute stage and chronic stage patients were not significant. Subfraction I was separated into three fractions (I-1, I-2, and I-3) by descending paper chromatography using solvent II, and subfraction III also was separated into three fractions (III-1, III-2, and III-3) by the same method. These 11 fractions of neutral oligosaccharides (I-1 through VII) were subjected to sequential exoglycosidase digestions, repeated Bio-Gel P-4 column chromatographies, methylation analysis, and GC/MS analysis. These analyses revealed the structures of oligosaccharides in these fractions as shown in Figure 2.

The monosialylated oligosaccharides were digested by $\alpha$-neuraminidase and were separated into seven subfractions (I through VII) by the Bio-Gel P-4 column chromatography (12) (Fig. 1), and the contents of each subfraction are shown in Table 3. Subfraction I was separated into two fractions (M-I-1, M-I-2) by descending paper chromatography using solvent II. The structures of monosialylated oligosaccharides were identified as shown in Figure 3 (M-I-1 through M-VII).

The disialylated oligosaccharides were separated into six subfractions by the Bio-Gel P-4 column chromatography (12) after $\alpha$-neuraminidase digestions (Fig. 1). The oligosaccharide contents of each subfraction are shown in Table 3 . The structures of disialylated oligosaccharides are shown in Figure 3 (D-II through D-VII).

Trisialylated oligosaccharides were separated into five subfractions by the Bio-Gel P-4 column chromatography (12) after $\alpha$ neuraminidase digestions (Fig. 1). The oligosaccharide contents of these five fractions are shown in Table 3. The structures of trisialylated oligosaccharides are shown in Figure 3 (T-III through T-VII)

These oligosaccharides (Figs. 2 and 3) were detected in the urine of all patients. Therefore, qualitative differences of urinary oligosaccharides were not found between the two clinical stages of the nephrotic syndrome.

Table 2. Urinary oligosaccharide content (nmol/mg creatinine) in nephrotic syndrome patients and galactosialidosis patients*

\begin{tabular}{ccccccc}
\hline & $\mathrm{T}$ & $\mathrm{N}$ & $\mathrm{S}$ & $\mathrm{MS}$ & $\mathrm{DS}$ & $\mathrm{TS}$ \\
\hline Nephrotic syndrome $(n=5)$ & $66.7 \pm 21.4$ & $34.3 \pm 11.1$ & $32.4 \pm 11.1$ & $7.9 \pm 3.2$ & $13.8 \pm 7.6$ & $10.7 \pm 4.8$ \\
Acute stage $(n=2)$ & $61.4 \pm 37.2$ & $28.3 \pm 15.6$ & $33.1 \pm 21.6$ & $8.1 \pm 5.9$ & $17.2 \pm 14.4$ & $7.8 \pm 1.3$ \\
Chronic stage $(n=3)$ & $70.3 \pm 13.4$ & $38.3 \pm 11.2$ & $32.0 \pm 8.7$ & $7.8 \pm 2.8$ & $11.6 \pm 4.7$ & $12.6 \pm 6.6$ \\
Galactosialidosis $(n=9) \dagger$ & $16.4 \pm 8.1$ & $4.7 \pm 2.4$ & $11.7 \pm 5.9$ & $8.4 \pm 5.4$ & $1.9 \pm 0.6$ & $1.4 \pm 0.8$ \\
Control $(n=6)$ & $2.6 \pm 0.5$ & $2.6 \pm 0.5$ & ND & &
\end{tabular}

* T, total oligosaccharides; N, neutral oligosaccharides; S, sialyl oligosaccharides: MS, monosialylated oligosaccharides; DS, disialylated oligosaccharides; TS, trisialylated oligosaccharides; ND, not detected.

† Data of urinary oligosaccharide contents in galactosialidosis patients are drawn from our previous paper (14). 

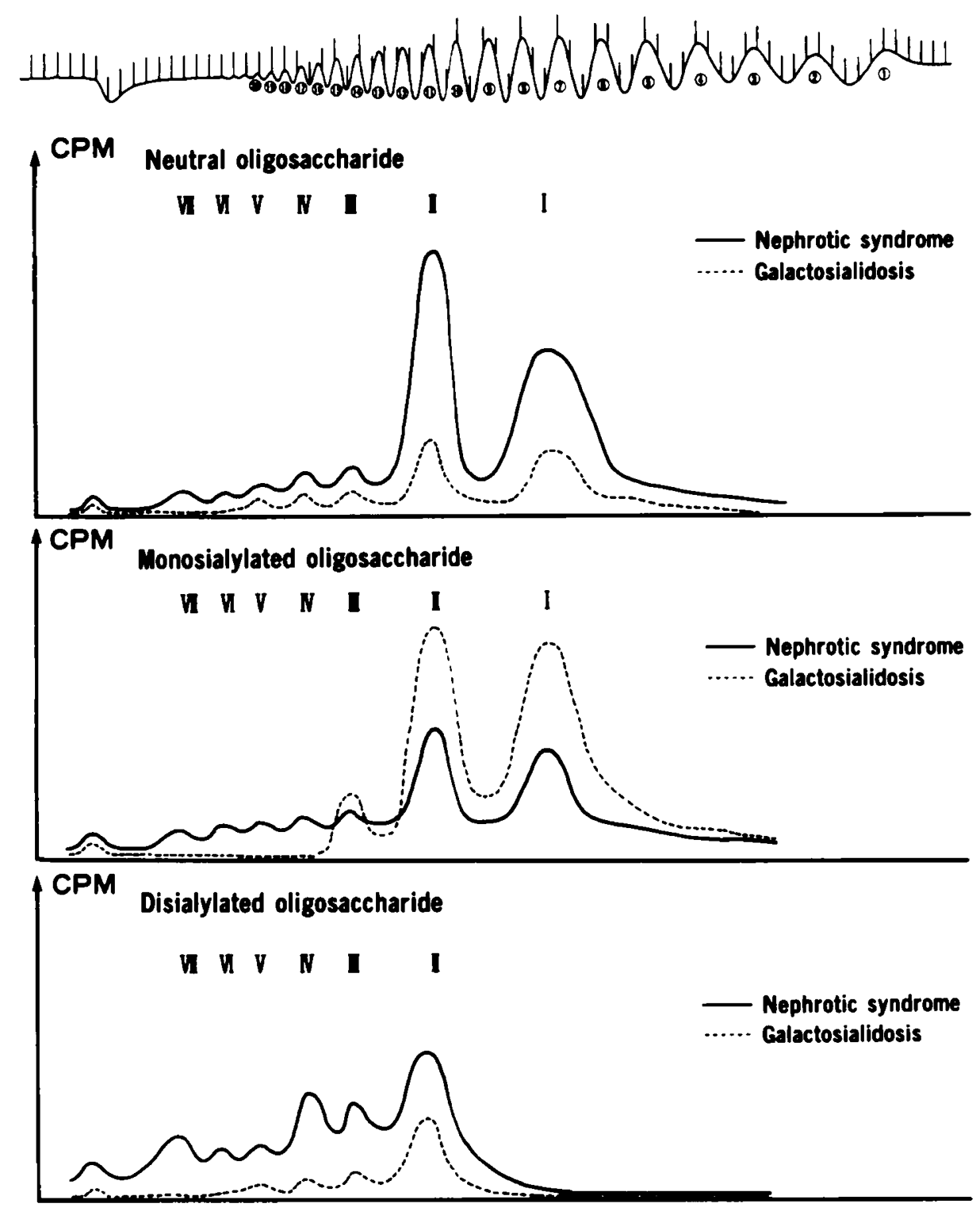

CPM Trisialylated oligosaccharide

ท ท $V$ N I

Nephrotic syndrome

Galactosialidosis

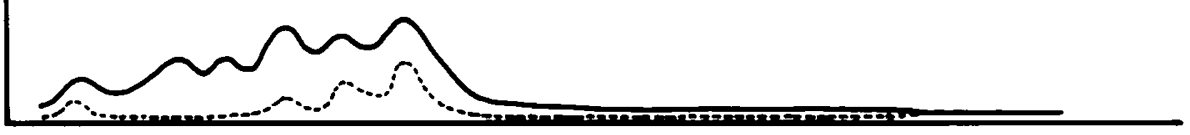

$\mathrm{I} \sim \mathrm{h}:$ fraction number

Fig. 1. Fractionation patterns of urinary oligosaccharides from nephrotic syndrome patients and galactosialidosis patients by means of Bio-Gel P-4 column chromatography under the conditions described in a previous paper (9). Circled numbers below the refractometer curves are glucose units that indicate the molecular size of oligosaccharide chains; i.e. oligosaccharides that are eluted at the position of 2 glucose units have twice the molecular size of glucose. I through VII are fraction numbers. Ordinates are radioactivities of oligosaccharide eluted at each position.

\section{DISCUSSION}

These patients were chosen sequentially in the limited periods without preference for data of urinary oligosaccharides. They have not manifested clinical symptoms that suggest hereditary neurologic diseases and have not run progressive unfavorable courses. Two acute stage patients have enjoyed remissions of nephrotic syndrome, and other chronic prolonged-stage patients have not shown worsening of clinical conditions and laboratory data. Therefore, these patients do not seem to suffer from oligosaccharidosis.

It is generally accepted that urinary oligosaccharides in oligosaccharidosis patients result from incomplete degradation of $N$ glycosidically linked oligosaccharides $(9,17)$. Further, we recently 
Table 3. Urinary oligosaccharide contents ( $\mathrm{mmol} / \mathrm{mg}$ creatinine) of each subfraction in nephrotic syndrome patients*

\begin{tabular}{lrrrrrrr}
\hline & I & II & III & IV & V & VI \\
\hline Neutral oligosaccharide $(n=5)$ & $13.8 \pm 7.4$ & $7.0 \pm 3.0$ & $4.0 \pm 1.4$ & $3.1 \pm 1.9$ & $2.1 \pm 1.4$ & $2.0 \pm 1.1$ & $2.2 \pm 1.3$ \\
Acute stage $(n=2)$ & $9.9 \pm 5.9$ & $7.9 \pm 4.7$ & $4.0 \pm 1.6$ & $2.5 \pm 1.6$ & $1.3 \pm 0.6$ & $1.4 \pm 0.8$ & $1.3 \pm 0.4$ \\
Chronic stage $(n=3)$ & $16.4 \pm 8.2$ & $6.4 \pm 2.5$ & $4.0 \pm 1.5$ & $3.5 \pm 2.3$ & $2.7 \pm 1.6$ & $2.4 \pm 1.3$ & $2.8 \pm 1.3$ \\
Monosialylated oligosaccharide $(n=5)$ & $1.9 \pm 0.6$ & $1.9 \pm 1.1$ & $1.2 \pm 0.6$ & $0.9 \pm 0.4$ & $0.6 \pm 0.3$ & $0.4 \pm 0.2$ & $0.9 \pm 0.5$ \\
Acute stage $(n=2)$ & $1.7 \pm 0.8$ & $2.2 \pm 1.7$ & $1.3 \pm 1.1$ & $1.0 \pm 0.8$ & $0.6 \pm 0.4$ & $0.4 \pm 0.2$ & $1.1 \pm 0.9$ \\
Chronic stage $(n=3)$ & $2.0 \pm 0.8$ & $1.0 \pm 1.0$ & $1.2 \pm 0.4$ & $0.8 \pm 0.3$ & $0.7 \pm 0.4$ & $0.5 \pm 0.2$ & $0.8 \pm 0.4$ \\
Disialylated oligosaccharide $(n=5)$ & ND & $3.1 \pm 1.4$ & $2.5 \pm 1.6$ & $2.9 \pm 2.0$ & $1.9 \pm 1.2$ & $1.4 \pm 0.8$ & $2.2 \pm 2.1$ \\
Acute stage $(n=2)$ & ND & $3.4 \pm 2.1$ & $3.2 \pm 2.3$ & $3.8 \pm 3.5$ & $2.5 \pm 2.1$ & $1.5 \pm 1.2$ & $3.0 \pm 3.3$ \\
Chronic stage $(n=3)$ & ND & $2.8 \pm 1.2$ & $2.0 \pm 1.3$ & $2.3 \pm 0.9$ & $1.5 \pm 0.6$ & $1.3 \pm 0.8$ & $1.6 \pm 1.5$ \\
Trisialylated oligosaccharide $(n=5)$ & ND & ND & $2.1 \pm 0.8$ & $1.9 \pm 0.5$ & $2.6 \pm 2.0$ & $1.2 \pm 0.9$ & $1.7 \pm 1.3$ \\
Acute stage $(n=2)$ & ND & ND & $1.9 \pm 0.3$ & $2.1 \pm 0.5$ & $1.7 \pm 0.1$ & $0.9 \pm 0.2$ & $1.3 \pm 0.4$ \\
Chronic stage $(n=3)$ & ND & ND & $2.3 \pm 1.3$ & $1.7 \pm 0.6$ & $3.5 \pm 3.0$ & $1.5 \pm 1.3$ & $2.1 \pm 2.1$ \\
\hline
\end{tabular}

* ND, not detected.

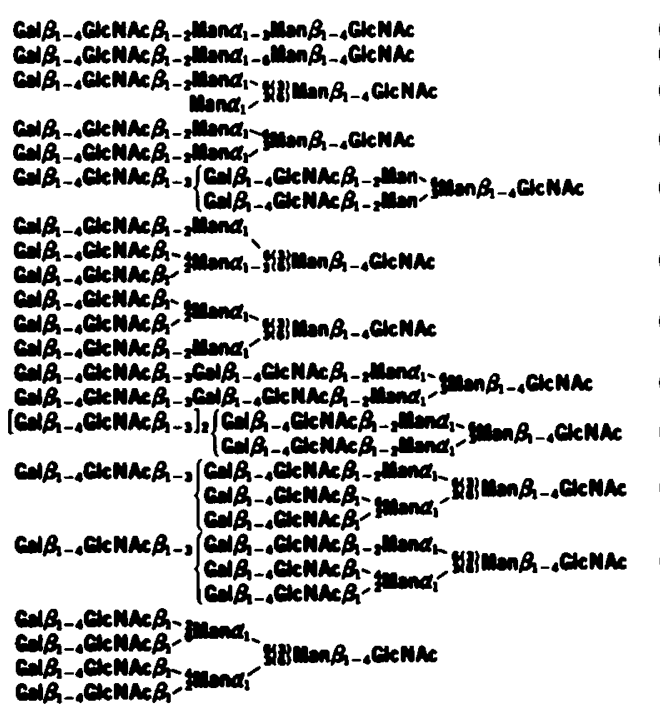

$\left(\begin{array}{c}(1-1) \\ (1-2)\end{array}\right.$

$(\mathbf{I}-3)$

(II)

(III-1)

(III-2)

(III-3)

(IV-1)

(IV-2)

$(\mathrm{IV}-3)$

$(I V-4)$

$(\mathrm{IV}-5)$

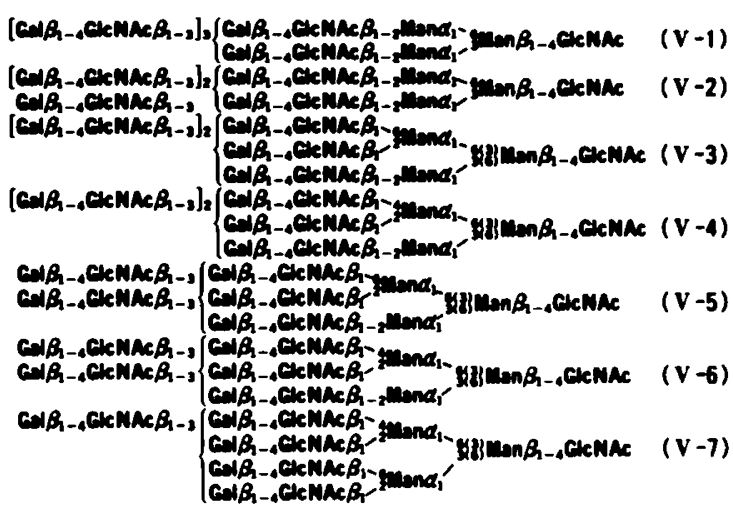

अ3): indicates ; and/or 3

$\left[\operatorname{Gol} \beta_{1-4} G i c N A c \beta_{1-3}\right]_{2}$ indicates $\left[G a \mid \beta_{1-4} \operatorname{GicNAC} \beta_{1}\right]-\left[{ }_{3} \operatorname{Gal} \beta_{1-4} \operatorname{GicNAC} \beta_{1-}\right]$

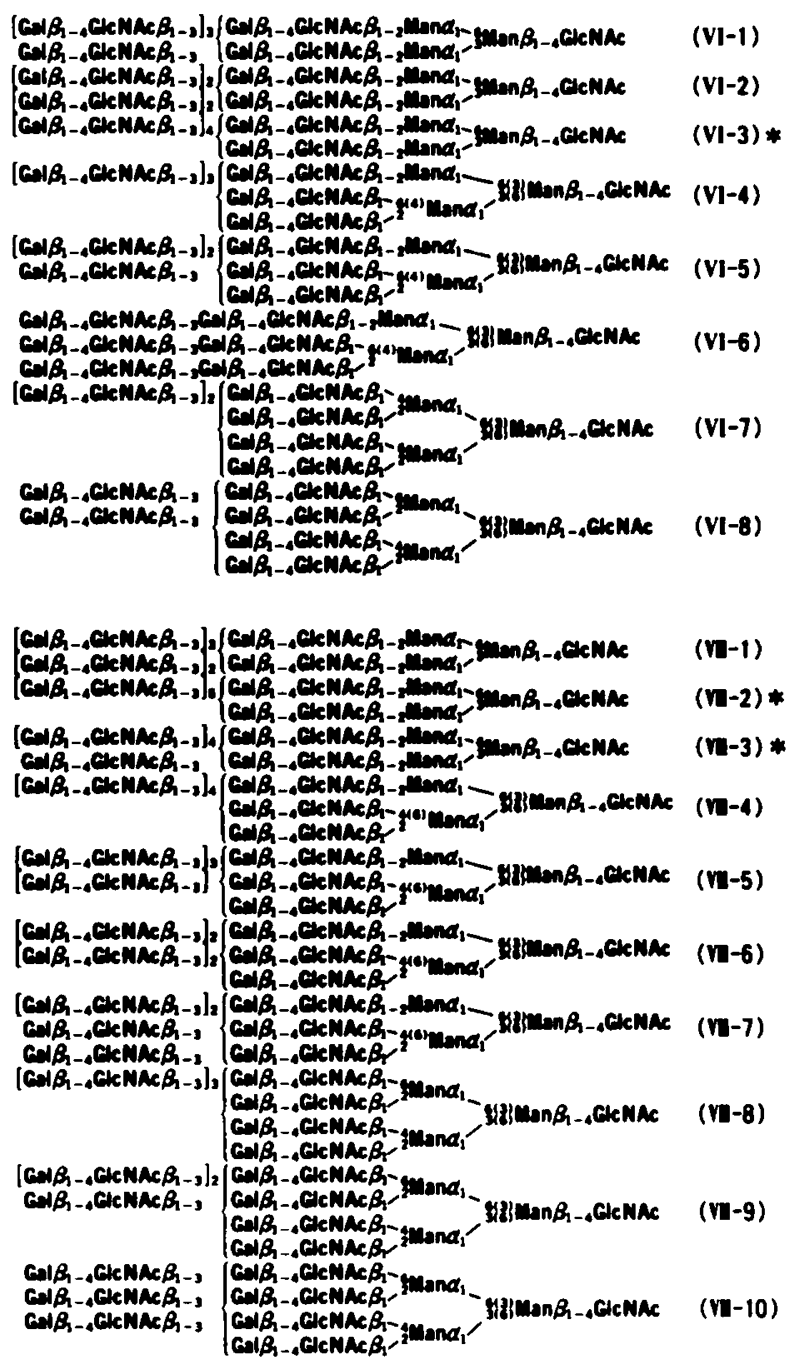

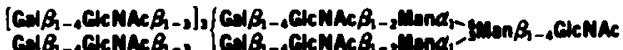

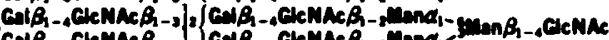

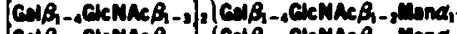

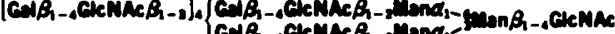

CN $\beta_{1}-4$ CeNA $\beta_{1}-2$ and

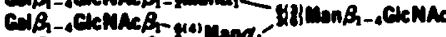

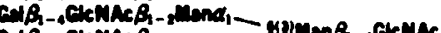

(V1-5)

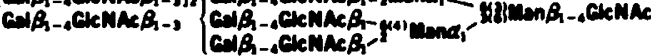

$(v 1-6)$

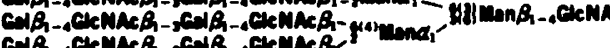

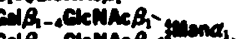

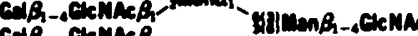

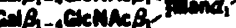

Col $\beta_{1}-4$ CleNAC $\beta_{1}$

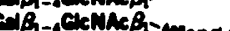

YHtrimen $\beta_{1-4}$ CleNAc

$-8)$

(v-1)

$(v-2)$ *

$(\mathrm{r}-3) *$

$(n-4)$

$(n-5)$

$(n-6)$

(vin-7)

( $(\mathrm{D}-8)$

(VI-9)

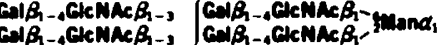

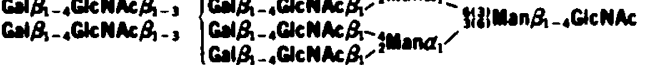

$(\mathrm{V}-10)$

Fig. 2. Proposed structures of neutral oligosaccharides excreted in the urine of nephrotic syndrome patients. [Gal $\left.\beta_{1-4} \mathrm{GlcNAc} \beta_{1-3}\right]_{2}$ indicates $\left[\mathrm{Gal} \beta_{1-4} \mathrm{GlcNAc} \beta_{1}\right]-\left[{ }_{3} \mathrm{Gal} \beta_{1-4} \mathrm{GlcNAc} \beta_{1-}\right]$. I through VII are fraction numbers on Bio-Gel P-4 column chromatography performed under the conditions described in a previous paper (9) (Fig. 1). Gal, galactose; GlcNAc, $N$-acetyl glucosamine; Man, mannose. *. Oligosaccharides not reported in previous papers.

reported that the same oligosaccharides accumulate in both the ascites and urine of an infantile sialidosis patient (18). This fact may indicate that possible accumulated oligosaccharides in the bloodstream, which originate from intracellular incomplete degradations, overflow to ascites and urine (18). Therefore, urinary oligosaccharides in oligosaccharidosis patients may reflect accumulated oligosaccharides in the bloodstream and may not result from the secretions of renal tubule cells.
This study revealed excessive excretions of galactosyl and sialyl oligosaccharides in the urine of acute and chronic nephrotic syndrome patients. Such oligosacchariduria, which is composed of galactosyl and sialyl oligosaccharides, was also reported in the urine of galactosialidosis patients in our previous research (19). The renal functions of our galactosialidosis patients were normal, and a coexistent deficiency of both $\alpha$-neuraminidase and $\beta$ galactosidase activities was the cause of galactosyl and sialyl 

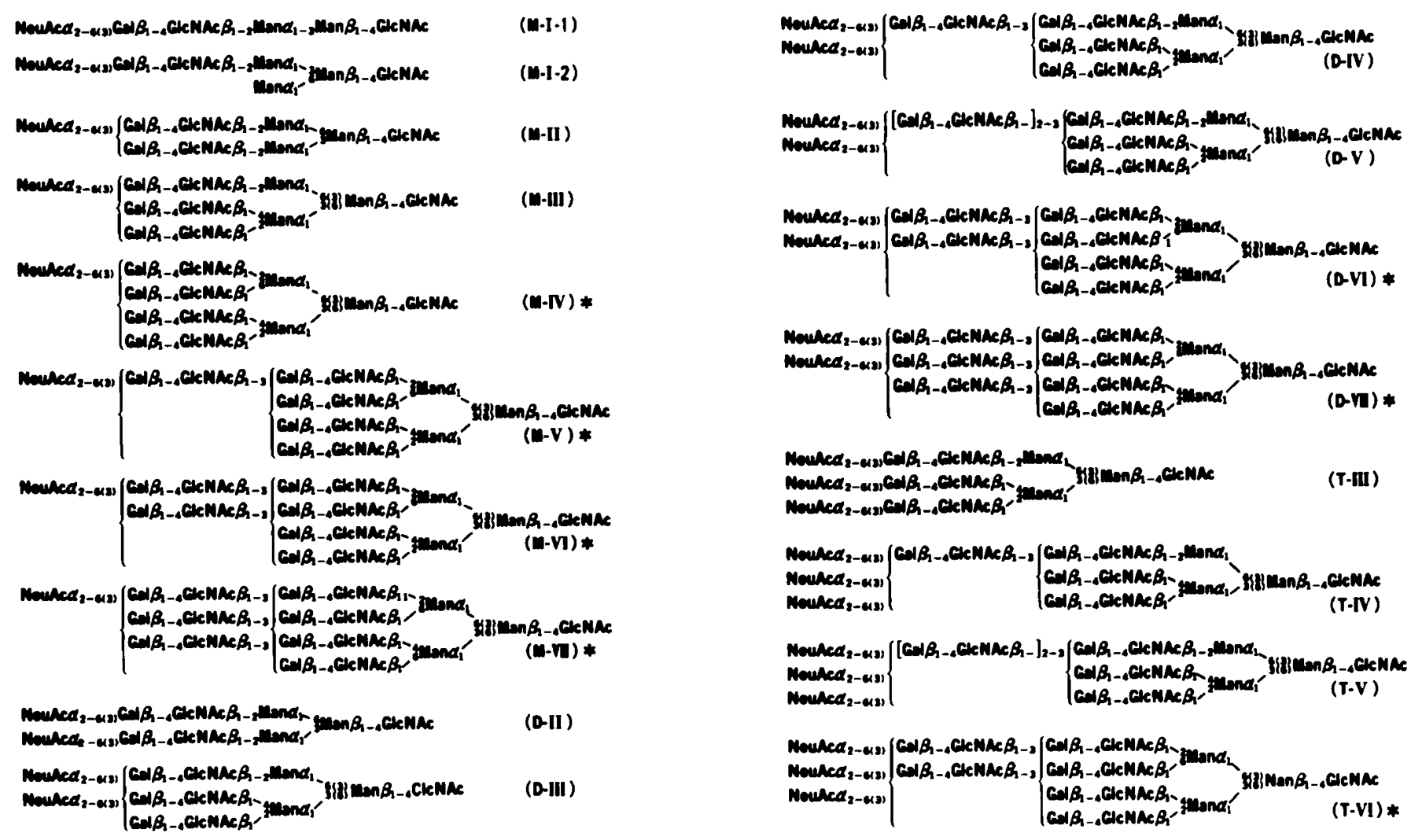

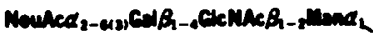

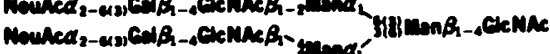

Conved
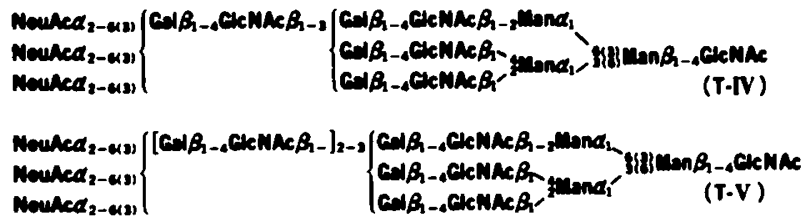

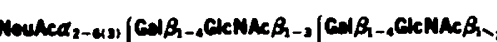

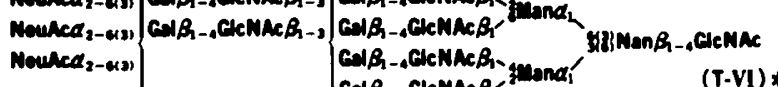

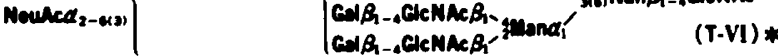

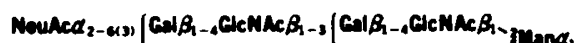

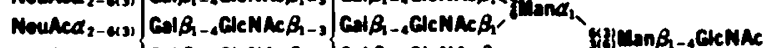

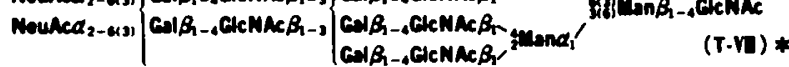

\section{6(3) indicates 6 and/or 3}

Fig. 3. Proposed structures of sialyl oligosaccharides excreted in the urine of nephrotic syndrome patients. [Gal $\left.\beta_{1-4} \mathrm{GlcNAc} \beta_{1-3}\right]_{2}$ indicates $\left[\mathrm{Gal} \beta_{1-4} \mathrm{GlcNAc} \beta_{1}\right]-\left[{ }_{3} \mathrm{Gal} \beta_{1-4} \mathrm{GlcNAc} \beta_{1-}\right]$. *, Oligosaccharides not reported in previous papers. Gal, galactose; $G l c N A c, N$-acetyl glucosamine; $M a n$, mannose; NeuAc, $N$-acetyl neuraminic acid.

oligosacchariduria. Thus, nephrotic syndrome patients with normal degradations of oligosaccharides and poor renal function excreted similar constituent oligosaccharides as galactosialidosis patients with incomplete degradations and normal renal function. To estimate the mechanisms of oligosacchariduria in nephrotic syndrome, we compared urinary oligosaccharides in nephrotic syndrome patients with those in galactosialidosis patients whose mechanisms of oligosacchariduria are considered as overflows of incompletely degradated oligosaccharides from the bloodstream (Table 2).

Nephrotic syndrome patients excreted four times as much oligosaccharide in their urine as did galactosialidosis patients. This quantitative difference in urinary oligosaccharides might suggest that the mechanisms that cause oligosaccharidurias are different between nephrotic syndrome and galactosialidosis.

The ratio of trisialylated oligosaccharides to monosialylated oligosaccharides in nephrotic syndrome patients $(1.68 \pm 1.11$, $n=5)$ was higher than that in galactosialidosis patients $(0.26 \pm$ $0.18, n=9$ ). Trisialylated oligosaccharides are more negatively charged than monosialylated oligosaccharides; therefore, these data indicate that the urine of nephrotic syndrome patients contains more highly negatively charged sialyl oligosaccharides than those of galactosialidosis patients. The predominance of highly negatively charged sialyl oligosaccharides in nephrotic syndrome patients suggests that electrostatic defects in the GBM may contribute to the sialyl oligosacchariduria in nephrotic syndrome.

The urinary contents of neutral noncharged oligosaccharides in nephrotic syndrome patients also exceed those in galactosiali- dosis patients. This implies that the defect of size barrier in the GBM also may contribute to the oligosacchariduria in nephrotic syndrome patients.

We cannot obtain enough serum to investigate oligosaccharides in the bloodstream of nephrotic syndrome patients and normal subjects, nor can we determine the serum levels of oligosaccharides. Therefore, we cannot conclude that the oligosacchariduria in nephrotic syndrome patients results exclusively from hyperpermeabilities derived from electrostatic defects and size barrier defects in the GBM. However, we can hardly agree with the idea that urinary accumulations of oligosaccharides in nephrotic syndrome are attributed to the increase of serum oligosaccharide levels for the following reasons. 1) Degradations of oligosaccharides in lysosomes, which supply mainly serum complex-type oligosaccharides, are considered to be normal in nephrotic syndrome. 2) The profiles of galactosyl and sialyl oligosaccharides in the urine of nephrotic syndrome patients differ from those in the urine of galactosialidosis patients whose mechanisms of oligosaccharidurias are considered as overflows from the bloodstream. Therefore, we have difficulty supporting the concept that increased oligosaccharides in the serum of a nephrotic syndrome patient explain fully the augmented urinary excretion.

It is well known that the renal tubules can reabsorb the glucose moiety almost completely but cannot reabsorb the dextran component. Conversely dynamics of complex-type oligosaccharide resorption in the human kidney are still unknown. Therefore, we cannot deny the possibility that the decreased tubular reabsorption of oligosaccharides might attribute to oligosacchariduria 
in nephrotic syndrome patients. However, we do not support this possibility for the following reasons. 1) Usual renal tubular diseases do not manifest oligosacchariduria. 2) Pathologic changes of MCNS patients are usually detected in the glomerulus not in the tubules. 3) The proteinuria of nephrotic syndrome is considered to result from the electrostatic defects and size barrier defects in the GBM.

From the above discussions, we estimate that the electrostatic defects and the size barrier defects in the GBM may contribute to the oligosacchariduria in nephrotic syndrome patients.

The ratios of neutral oligosaccharides to sialyl oligosaccharides in the urine of nephrotic syndrome patients $(1.11 \pm 0.41, n=5)$ were higher than those in the urine of galactosialidosis patients $(0.41 \pm 0.08, n=9)$. In galactosialidosis patients, the deficiency of $\alpha$-neuraminidase is more severe than the deficiency of $\beta$ galactosidase, so the accumulations of sialyl oligosaccharides seem to exceed the accumulation of galactosyl oligosaccharides in the organs and the urine. In nephrotic syndrome patients, defects of the electrostatic barrier and size barrier in the GBM may contribute to harmonious urinary excretions of galactosyl oligosaccharides and sialyl oligosaccharides. Conclusive proof of this hypothesis awaits further experimental evidence.

Last, the quantitative and qualitative differences of urinary oligosaccharides between acute stage and chronic stage patients were not significant; and the structures of three previously unreported kinds of galactosyl oligosaccharides and eight previously unreported kinds of sialyl oligosaccharides were determined (VI3, VII-2, VII-3, M-IV, M-V, M-VI, M-VII, D-VI, D-VII, T-VI, T-VII).

Acknowledgments. The authors thank Dr. A. Sakai, Dr. K. Gotou, and Dr. T. Kuwahara for urine sample donations; K. Sukegawa for technical advice; and M. Sekiya and T. Kameyama for skillful technical assistance.

\section{REFERENCES}

1. Karynovsky MJ. Ainsworth SK 1972 Advances in Nephrology, Vol 2. Year Book Medical Publishers, Chicago, pp 35-60
2. Farquhar MG 1975 The primary glomerular filtration barrier: basement membrane or epithelial slits? Kidney Int 8:197-211

3. Kounou M. Itou K 1990 Structures of GBM and urinary protein: concentrated on protein losing mechanisms (Japanese title). Shouni Naika 22:725-729

4. Brenner BM, Hostetter TH, Humes HD 1978 Molecular basis of proteinuria of glomerular origin. N Engl J Med 298:828-833

5. Bohrer MP. Baylis C, Humes HD, Glassock RJ 1978 Permselectivity of the glomerular capillary wall. J Clin Invest 61:72-78

6. Carrie BJ, Salyer WR, Myers DB 1981 Minimal change nephropathy: an electrochemical disorder of the glomerular membrane. Am J Med 70:262268

7. Bridges CR, Myers BD. Brenner BM. Deen WM 1982 Glomerular charge alterations in human minimal change nephropathy. Kidney lnt 22:677-684

8. Kanwar YS, Linker A, Farquhar MG 1980 Increased permeability of the glomerular basement membrane to ferritin after removal of glycosaminoglycans (heparan sulfate) by enzyme digestion. J Cell Biol 86:688-693

9. Beaudet AL. Thomas GH 1989 Disorders of glycoprotein degradation: mannosidosis. fucosidosis. sialidosis, and aspartylglycosaminuria. In: Stanbury JB, Wyngaarden JB, Fredrickson DS, (eds) The Metabolic Basis of Inherited Disease. McGraw-Hill Book Company, New York, pp 1603-1621

10. Sewell AC 1980 Urinary oligosaccharide excretion in disorders of glycolipid. glycoprotein and glycogen metabolism: a review of screening for differential diagnosis. Eur J Pediatr 134:183-194

11. Durand P. Gatti R, Cavalieri S, Borrone C. Tondeur M, Michalski JC. Strecker G 1977 Sialidosis (mucolipidosis I). Helv Paediatr Acta 2:391-400

12. Nishigaki M. Yamashita K. Matsuda I. Arashima S. Kobata A 1978 Urinary oligosaccharides of fucosidosis. J Biochem 84:823-834

13. Takahashi $Y$. Orii $T 1989$ Severity of $G_{M}$ gangliosidosis and urinary oligosaccharide excretion. Clin Chim Acta 179:153-162

14. Takasaki S. Kobata A 1974 Microdetermination of individual neutral and amino sugars and $N$-acetylneuraminic acid in complex saccharides. J Biochem 76:783-789

15. Yamashita K. Ohkura T, Okada S. Yabuuchi H, Kobata A 1981 Urinary oligosaccharides of $G_{M 1}$ gangliosidosis. J Biol Chem 256:4789-4798

16. Endo Y. Yamashita K. Tachibana Y. Tojo S, Kobata A 1979 Structures of asparagine-linked sugar chains of human chorionic gonadotropin. J Biochem 85:669-679

17. Warner TG, Robertson AD. O'Brien JS 1983 Diagnosis of $G_{M}$ gangliosidosis based on detection of urinary oligosaccharides with high performance liquid chromatography. Clin Chim Acta 127:313-326

18. Nakamura Y, Takahashi Y. Yamaguchi S, Omiya S, Orii T, Yara A. Gushiken M 1992 Severe infantile sialidosis: the characteristics of oligosaccharides isolated from the urine and the abdominal ascites. Tohoku J Exp Med 166:407-415

19. Takahashi Y, Nakamura Y, Yamaguchi S, Orii T 1991 Urinary oligosaccharide excretion and severity of galactosialidosis and sialidosis. Clin Chim Acta 203:199-210 